\title{
Simulación numérica 2D subrasantes de baja resistencia, estabilizadas con material granular de sobretamaño (rajón)
}

\section{Low resistance subgrade 2D numerical simulation stabilized with oversized granular material (boulders)}

\author{
Edwar Basante-Bolaños ${ }^{1 a}$, Miguel Villacrés-Martínez ${ }^{1 b}$, \\ Lucio Cruz-Velasco ${ }^{1 c}$ \\ ${ }^{1}$ Geotecnia Vial y Pavimentos, Geotecnia, Universidad del Cauca, Colombia. Correos electrónicos: \\ a edwarbasante@unicauca.edu.co, ${ }^{\mathrm{b}}$ mvillacrs@gmail.com, ${ }^{\mathrm{c}}$ lucruz@unicauca.edu.co \\ Orcid: ${ }^{\mathrm{c}} 0000-0003-2438-5526$
}

Recibido: 16 septiembre, 2020. Aceptado: 18 enero, 2021. Versión final: 5 mayo, 2021.

\begin{abstract}
Resumen
Dentro de las alternativas de estabilización de subrasantes existe tradicionalmente el mejoramiento con material de sobretamaño o rajón, tamaño aproximado de 10 pulgadas. El rajón forma un esqueleto mineral grueso que soporta la estructura y favorece el control de humedad. Esta técnica no tiene un protocolo de diseño establecido actualmente en Colombia. Esta investigación evalúa mediante simulación numérica elástica $2 \mathrm{D}$, y de manera cuantitativa, el aumento de propiedades mecánicas del conjunto del pavimento, mediante el mejoramiento de subrasantes blandas con material de sobretamaño (rajón). A partir de las modelaciones numéricas 2D se realizan comparativas de comportamiento y análisis de los resultados de variables fundamentales en el diseño de pavimentos. De esta manera, la investigación proporciona lineamientos para un protocolo de diseño para el mejoramiento de propiedades mecánicas de subrasantes blandas, mediante el mejoramiento de las mismas con material de sobretamaño.
\end{abstract}

Palabras clave: material de sobretamaño; modelación numérica; pavimento; rajón; subrasante; estabilización; mejoramiento; estructura; diseño; deformación unitaria; desplazamiento; esfuerzo.

\begin{abstract}
Among several subgrade stabilization alternatives, the improvement with oversized material (boulders of approximately 10-inches in size) has traditionally been used. The Boulders form a thick mineral skeleton that supports the structure and favors moisture control. Nowadays, this technique is not included within the design protocols of Colombia. In this research, the mechanical properties of the pavement are quantitatively evaluated through 2Dnumerical-elastic modeling employing the improvement of soft subgrades with boulders. Comparative studies of performance and analysis of the fundamental variables in pavement design are carried out through 2D-numerical modeling. This way, guidelines to sketch a design protocol are suggested to improve mechanical properties in soft subgrades through the inclusion of oversized material.
\end{abstract}

ISSN impreso: 1657 - 4583. ISSN en línea: 2145 - 8456, CC BY-ND 4.0 @) (1) @

Como citar: E. Basante-Bolaños, M. Villacrés-Martínez, L. Cruz-Velasco, "Simulación numérica 2D subrasantes de baja resistencia, estabilizadas con material granular de sobretamaño (rajón)," Rev. UIS Ing., vol. 20, no. 3, pp. 61-76, 2021, doi: $\underline{10.18273 / \text { revuin.v20n3-2021004 }}$ 
Keywords: boulder; numerical simulation; oversize material; pavement; subgrade; stabilization; improvement; structure; design; strain; displacement; stress.

\section{Introducción}

El mejoramiento de subrasantes con bajas propiedades mecánicas es tratado con diversas tecnologías, entre las que se encuentran los tratamientos físicos, químicos y/o mecánicos. Estos procedimientos aportan características estructurales mediante la adición de cementantes o mediante la utilización de geosintéticos, los cuales cuentan con parámetros de diseño establecidos. Una alternativa de carácter físico para mejorar la resistencia mecánica del suelo, y que constituye el tema de este trabajo, es el tratamiento de subrasantes de mala calidad con adición de material de sobretamaño o rajón (tamaño aproximado de 10 pulgadas) (véase figura 1). El rajón forma un esqueleto mineral grueso que soporta la estructura y favorece el control de humedad, debido a los espacios entre conglomerados.

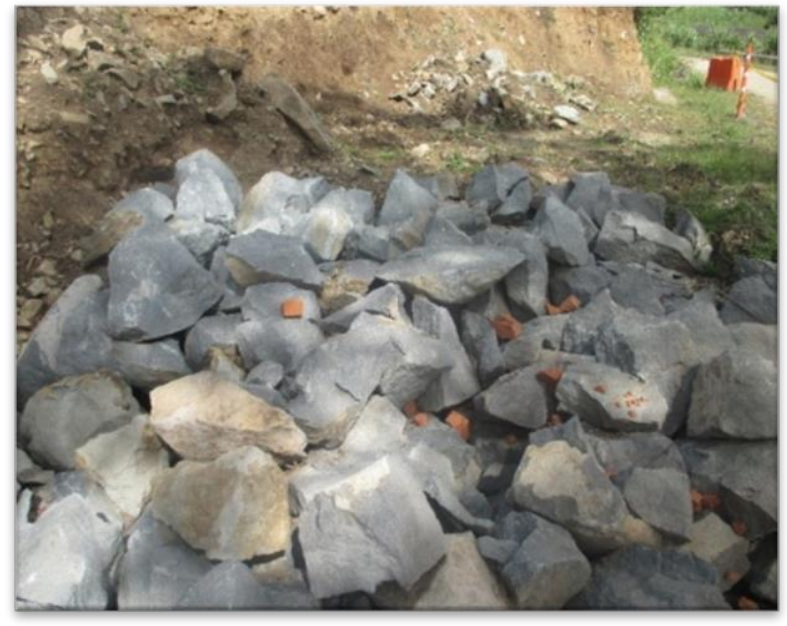

Figura 1. Fotografía de rajón utilizado para estabilización de suelos blandos. Fuente: elaboración propia.

Entre las muchas consideraciones a tener en cuenta para determinar una subrasante blanda o de baja calidad se tiene las siguientes: el Manual de diseño de pavimentos asfálticos para vías con bajos volúmenes de tránsito [1] categoriza como "malo" el comportamiento de una subrasante con CBR menor o igual a tres por ciento (CBR $\leq 3 \%$ ). De igual manera, si el suelo cuenta con un valor de resistencia al corte entre treinta y noventa kilopascales (30 kPa-90 kPa) también requiere estabilización; para el estudio de esta propiedad se sigue la norma INV E-152 "Compresión inconfinada en muestras de suelos" o INV E-170 "Ensayo de corte sobre suelos cohesivos, usando la veleta", [2].
El uso de insertos (rajón) se encuentra contemplado en normas nacionales: normas y especificaciones de Invías, en su Artículo 230-13 [2], y, de manera similar, el Instituto de Desarrollo Urbano de la Alcaldía Mayor de Bogotá D. C., en sus especificaciones de construcción, contempla un mejoramiento de subrasante, específicamente, con rajón [3].

Este mejoramiento se realiza en presencia de subrasantes blandas con capacidades de soporte bajas, generalmente, con CBR inferiores a 2,5\% en condición de humedad natural o 1,5\% en condición sumergida, o cuando se encuentren rellenos y/o suelos indeseables que sea necesario reemplazar, con el fin de mitigar problemas de hundimientos y/o de deformaciones plásticas.

Sin embargo, en la actualidad se da importancia a la experiencia del ingeniero a cargo y a bases empíricas para la toma de decisiones, dejando en evidencia que no hay lineamientos o pautas de carácter racional basados en investigación.

Como material principal de este tipo de estabilización denominado rajón, el Manual de especificaciones técnicas de diseño y construcción de parques y escenarios públicos de Bogotá D. C. [4] menciona que debe proceder de fuentes autorizadas y deberán ser fragmentos angulares de roca sana, compactas, resistentes y durables; preferiblemente areniscas duras, cuarzosas. Para el resto del país podrían utilizarse fragmentos de roca equivalentes.

En este trabajo se realizó un análisis mediante modelación numérica (bajo el modelo elástico lineal) de la estructura con y sin rajón, y se identificaron los efectos en la estructura en cuanto a desplazamientos, esfuerzos y deformaciones sobre las distintas capas del pavimento (subrasante, subbase, base y capa de rodadura). Adicionalmente, se evaluó mediante la simulación numérica elástica, y de manera cuantitativa, el mejoramiento de propiedades mecánicas de subrasante, $\mathrm{y}$, por consiguiente, subbase, base y carpeta de una estructura de pavimento flexible típica de la zona del suroccidente de Colombia, mediante el mejoramiento de la subrasante con material de sobretamaño (rajón). Como resultado se obtuvieron lineamientos mínimos para un protocolo de diseño que busque el mejoramiento de propiedades mecánicas de subrasantes blandas mediante la adición de material de sobretamaño (rajón), utilizando herramientas de modelación numérica. 


\section{Metodología}

Se identificaron los distintos métodos para la estabilización de una subrasante blanda, seleccionando los más utilizados en el medio [5], [6], [7], y, en específico, la estabilización de subrasante mediante la adición de material granular de sobretamaño o rajón [8].

Se consultó la normativa a nivel nacional tanto del Instituto Nacional de Vías como del Instituto de Desarrollo Urbano IDU [2], [4], los cuales dentro de sus normas y/o especificaciones plantean las actividades a llevar a cabo para la utilización de materiales de sobretamaño.

Con respecto a la obtención de datos como esfuerzos y/o deformaciones necesarias para el diseño de la estructura de pavimento, los distintos softwares de diseño de pavimentos solicitan áreas uniformes e isotrópicas para llevar a cabo el procedimiento [10] lo cual no se logra con la utilización del rajón embebido en la estructura [11]. Por lo tanto, se optó por el software de simulación ABAQUS [12].

\subsection{Tipo de estructura de pavimento propuesta para modelación}

Para la simulación de mejoramiento de estructuras de pavimento, se utilizó subrasantes de baja capacidad mecánica [13] $(\mathrm{CBR} \leq 3 \%)$, pero adicionalmente se adoptó, para la selección de la estructura de pavimento tipo, una que se enmarcaría en un trazado de una vía secundaria, con bajo tránsito, típica del departamento de Nariño o del departamento del Cauca, hacia la parte noroccidental y oriental de estos departamentos, respectivamente.

La estructura de pavimento a modelar en el presente trabajo está conforme con la metodología AASHTO (1993) [14].

Dado el bajo nivel de tránsito, y considerando las limitaciones de tipo operativo durante la etapa de construcción y mantenimiento, se optó por un periodo de diseño de diez (10) años [15].

Los métodos usuales para el diseño de pavimentos asfálticos consideran el nivel de tránsito en términos de repeticiones de ejes de $80 \mathrm{kN}$ en el carril de diseño. Para efectos del presente estudio se considera un nivel de tránsito menor a 500.000 ejes [16].
De acuerdo con la localización de la estructura a analizar, su clima asociado, y de acuerdo con el índice de Thornthwaite, se determinó que se trata de un clima "superhúmedo" [1]. Teniendo en cuenta el Manual de diseño de pavimentos asfálticos para vías con bajos volúmenes de tránsito del Invías [1], se determinó, como la estructura de pavimento de análisis, la siguiente:

- Alternativa 5: MDC-2 + BG + SBG (alternativa tomada para efectos del presente estudio).

Donde:

- TSD: tratamiento superficial doble

- BG: base granular

- SBG: subbase granular

- BEC: base estabilizada con cemento portland

- MDF-2: mezcla densa en frío tipo 2

- BEE-3: suelo estabilizado con emulsión.

El espesor mínimo establecido por el método AASHTO93 en función de la categoría de tráfico (menor a 500.000 ejes de $80 \mathrm{kN}$ ) corresponde a $75 \mathrm{~mm}$.

Teniendo en cuenta todo lo anterior, la estructura típica a modelar sería la indicada en la figura 2 .

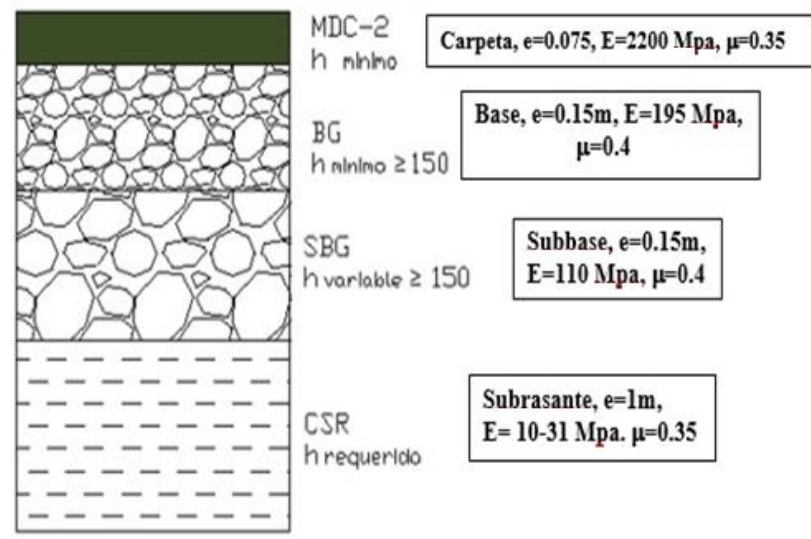

Figura 2. Estructura modelo. Fuente: adaptación propia de [1].

\subsection{Modelación en ABAQUS de estructura de pavimento propuesta}

\subsubsection{Propiedades de los materiales}

Las propiedades de los materiales propuestos para las distintas capas de la estructura de pavimento se supeditan en sus módulos de elasticidad y módulo de Poisson, los cuales son valores típicos en el medio [17, 18]. Incluyendo el módulo del rajón, de la siguiente manera: 
- Subrasante: 10, 20, $31 \mathrm{Mpa}$ (según sea el caso)

- Subbase: $110 \mathrm{Mpa}$

- Base: $195 \mathrm{Mpa}$

- Concreto asfáltico: $2200 \mathrm{Mpa}$

- Rajón: 68.000 Mpa (zona de roca sana).

Los anteriores valores son módulos típicos obtenidos de diferentes formulaciones o métodos. Los módulos de Poisson son valores típicos para los diferentes materiales de la estructura de pavimento (véase la figura 2).

\subsubsection{Interacción entre las partes del modelo}

En primera instancia, se crean y especifican las diferentes propiedades de contacto que controlan el modelo entre sus partes. Como primera propiedad se determinó un comportamiento tangencial de carácter "rígido"; como segunda propiedad se determinó un "comportamiento normal" bajo las características de "fuerte contacto", y como método de aplicación de restricciones, "por defecto".

\subsubsection{Aplicación de cargas}

En el presente caso se utilizaron cargas distribuidas aplicadas en la parte superior central del modelo de estructura de pavimento establecido, representando la carga de dos llantas correspondientes a un eje dual, cada una con $550 \mathrm{kPa}$ de presión en una huella de a=0,108 m de radio y separación c-c 3a, eje típico AASHTO. También, en esté módulo se determinan las condiciones o restricciones de bordo en lado inferior y en los dos lados laterales, para un mejor control de la estructura.

\subsubsection{Mallado}

Para cada parte del modelo se utilizaron elementos cuadrilateros, espaciados cada $0,10 \mathrm{~m}$, a excepción de la carpeta asfáltica, la cual espació los elementos cada $0,025 \mathrm{~m}$.

\subsubsection{Análisis de datos}

Se visualizan y se obtienen los resultados especificados mediante un reporte, que para el caso fueron: deformaciones (E11, E22), esfuerzos (S11, S22) y desplazamientos (U11, U22). Dirección 11 se refiere a eje horizontal X y dirección 22 se refiere a eje vertical Y.

\section{Resultados}

\subsection{Modelaciones - estructura sin rajón}

En la tabla 1 se presentan las diferentes modelaciones a realizadas.
Tabla 1. Modelaciones base

\begin{tabular}{|c|c|c|}
\hline \multicolumn{3}{|c|}{ Modelaciones base } \\
\hline $\begin{array}{c}\text { N. }{ }^{\circ} \text { de } \\
\text { modelación }\end{array}$ & CBR \% & $\begin{array}{c}\text { Diametro de } \\
\text { rajón }\end{array}$ \\
\hline 1 & 1 & Sin rajón \\
\hline 2 & 2 & Sin rajón \\
\hline 3 & 3 & Sin rajón \\
\hline
\end{tabular}

Fuente: elaboración propia.

\subsection{Consideraciones del modelo}

De acuerdo con el diseño planteado, se trabaja una estructura de pavimento con las siguientes características (véase tabla 2):

Tabla 2. Estructura sin mejoramiento

\begin{tabular}{|l|c|c|c|c|}
\hline \multicolumn{1}{|c|}{ Capa } & $\begin{array}{c}\text { Ancho } \\
(\mathbf{m})\end{array}$ & $\begin{array}{c}\text { Espesores } \\
(\mathbf{m})\end{array}$ & $\mathbf{E}[\mathbf{M P a}]$ & $\mathbf{v}$ \\
\hline Carpeta & 3 & 0,075 & 2200 & 0,35 \\
\hline Base & 3 & 0,15 & 195 & 0,4 \\
\hline Subbase & 3 & 0,15 & 110 & 0,4 \\
\hline Subrasante & 3 & 1 & $\begin{array}{c}\text { Depende } \\
\text { CBR }\end{array}$ & 0,45 \\
\hline
\end{tabular}

Fuente: elaboración propia.

Se realiza un análisis de desplazamientos, esfuerzos y deformaciones unitarias a lo largo del eje " $X$ " (horizontal), correspondiente a la parte inferior de la carpeta asfáltica, en la subrasante a nivel de $1 \mathrm{~m}$ y $0,7 \mathrm{~m}$; también se annaliza el eje "Y" (vertical) que atraviesa el centro de la estructura de pavimento propuesta (nodos rojos) (véase figura 4).

Teniendo en cuenta lo anterior, las coordenadas globales del origen tanto del eje "X" y "Y" son: $1,5 \mathrm{~m}$ y $0,0 \mathrm{~m}$ (Véanse figura 3 y figura 4 ).

\subsection{Modelaciones ejecutadas - estructuras con diferentes configuraciones de rajón}

Una vez ejecutadas las modelaciones base para las estructuras de pavimento con diferente tipo de subrasante (1\%, $2 \%$ y $3 \%$ de CBR), se procedió a ejecutar las diferentes modelaciones con las estructuras de pavimento, mejorando la subrasante con rajón (veáse tabla 3). 


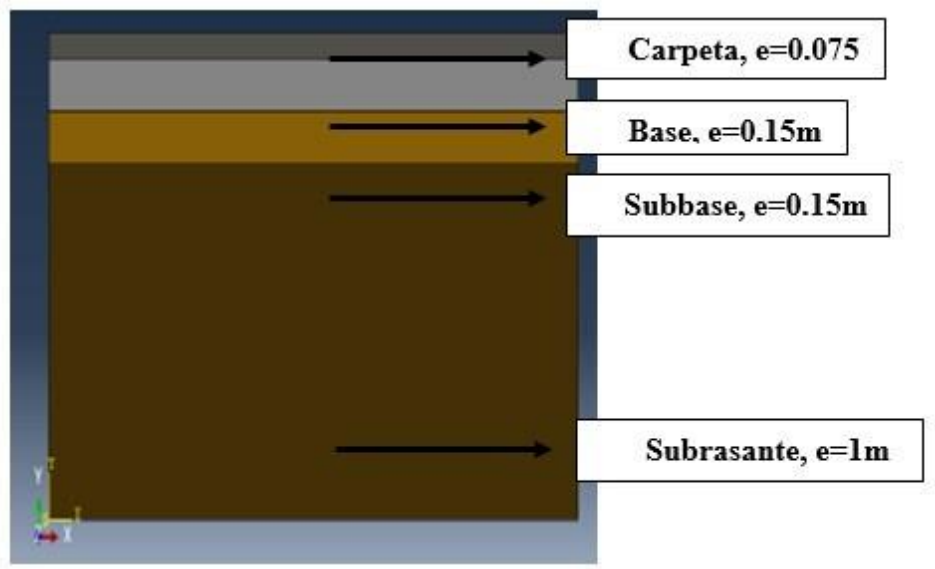

Figura 3. Estructura de pavimento en software Abaqus. Fuente: elaboración propia.

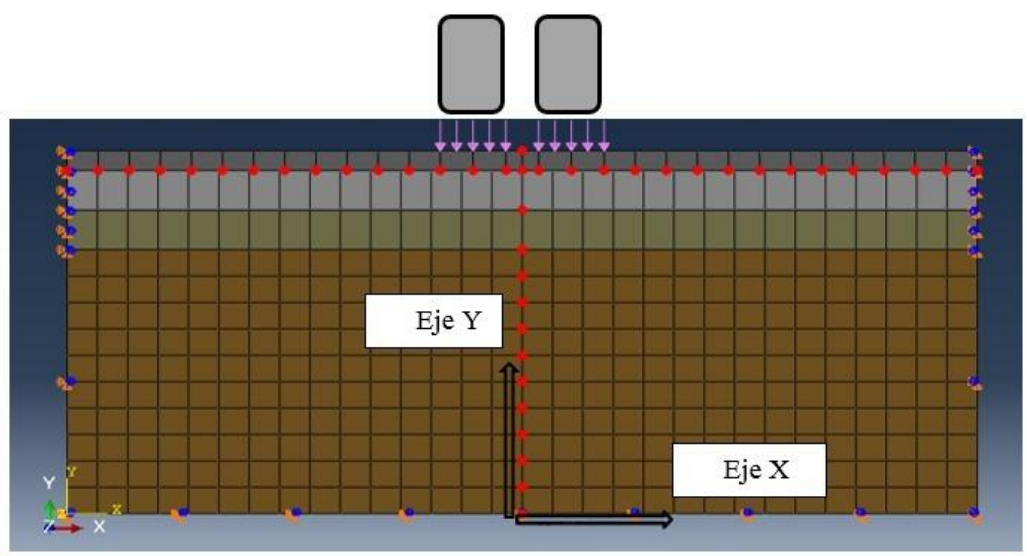

Figura 4. Origen ejes de análisis. Fuente: elaboración propia.

Inicialmente, se modelaron las estructuras de pavimento con los CBR de $1 \%, 2 \%$ y $3 \%$, las cuales son las modelaciones de referencia o base para determinar cómo el rajón cambia el comportamiento de la estructura. Luego, se realizaron modelaciones numéricas con el rajón y se probaron diferentes configuraciones de colocación del rajón, entre ellas: diámetro de la partícula, separación de las partículas (separación entre bordes externos de rajón), penetración de las partículas en la capa de subrasante (porcentaje de penetración de rajón en subrasante respecto a su diámetro) y número de filas colocadas.

En total, se trata de 42 modelaciones, de las cuales se obtuviron 6 diagramas de contorno para cada una, para un total de 252 diagramas de contorno correspondientes a desplazamiento, deformación unitaria y esfuerzos; además, por cada modelación se obtuvieron 18 gráficas del comportamiento de los parámetros mencionados, es decir, que en total se tienen $\mathbf{7 5 6}$ gráficas correspondientes a desplazamiento, deformación unitaria y esfuerzos. En la tabla 3 se presentan las modelaciones ejecutadas.

A continuación, se presenta la modelación $n .^{\circ}$ 36. (véanse figura 5-28). Para esta modelación se utilizaron los siguientes parámetros: CBR $1 \%$, diámetro del rajón $25 \mathrm{~cm}$, separación $0 \mathrm{~m}$, penetración del rajón en la capa subrasante al 50 \% y 3 filas de rajón. 
Tabla 3. Modelaciones estructuras de pavimento con mejoramiento en rajón para las subrasantes

\begin{tabular}{|c|c|c|c|c|c|}
\hline \multicolumn{6}{|c|}{ MODELACIONES A EJECUTAR } \\
\hline CBR (\%) & $\begin{array}{c}\text { No DE } \\
\text { MODELACIÓN }\end{array}$ & $\begin{array}{l}\text { DIÁMETRO } \\
\text { DE RAJON }\end{array}$ & $\begin{array}{c}\text { SEPARACIÓN } \\
\text { ENTRE } \\
\text { BORDES }(\mathrm{m})\end{array}$ & $\begin{array}{l}\text { PENETRACIÓN EN } \\
\text { SUBRASANTE (\%) }\end{array}$ & No DE FILAS \\
\hline \multicolumn{6}{|c|}{ PARAMETRIZAR DIAMETRO DE RAJÓN CON RESPECTO AL ESPESOR DE LA CARPETA } \\
\hline \multirow{3}{*}{1} & 4 & 0.25 & 0 & 50 & 1 \\
\hline & 5 & 0.15 & 0 & 50 & 1 \\
\hline & 6 & 0.35 & 0 & 50 & 1 \\
\hline \multirow{3}{*}{2} & 7 & 0.25 & 0 & 50 & 1 \\
\hline & 8 & 0.15 & 0 & 50 & 1 \\
\hline & 9 & 0.35 & 0 & 50 & 1 \\
\hline \multirow{3}{*}{3} & 10 & 0.25 & 0 & 50 & 1 \\
\hline & 11 & 0.15 & 0 & 50 & 1 \\
\hline & 12 & 0.35 & 0 & 50 & 1 \\
\hline \multicolumn{6}{|c|}{ PARAMETRIZAR ESPACIAMIENTO DE RAJÓN CON RESPECTO AL ESPESOR DE LA CARPETA } \\
\hline \multirow{4}{*}{1} & 13 & 0.25 & 0 & 50 & 1 \\
\hline & 14 & 0.25 & 0.0625 & 50 & 1 \\
\hline & 15 & 0.25 & 0.125 & 50 & 1 \\
\hline & 16 & 0.25 & 0.25 & 50 & 1 \\
\hline \multirow{4}{*}{2} & 17 & 0.25 & 0 & 50 & 1 \\
\hline & 18 & 0.25 & 0.0625 & 50 & 1 \\
\hline & 19 & 0.25 & 0.125 & 50 & 1 \\
\hline & 20 & 0.25 & 0.25 & 50 & 1 \\
\hline \multirow{4}{*}{3} & 21 & 0.25 & 0 & 50 & 1 \\
\hline & 22 & 0.25 & 0.0625 & 50 & 1 \\
\hline & 23 & 0.25 & 0.125 & 50 & 1 \\
\hline & 24 & 0.25 & 0.25 & 50 & 1 \\
\hline \multicolumn{6}{|c|}{ PARAMETRIZAR PENETRACIÓN DE RAJÓN CON RESPECTO A SU DIAMETRO } \\
\hline \multirow{3}{*}{1} & 25 & 0.25 & 0 & 25 & 1 \\
\hline & 26 & 0.25 & 0 & 50 & 1 \\
\hline & 27 & 0.25 & 0 & 75 & 1 \\
\hline \multirow{3}{*}{2} & 28 & 0.25 & 0 & 25 & 1 \\
\hline & 29 & 0.25 & 0 & 50 & 1 \\
\hline & 30 & 0.25 & 0 & 75 & 1 \\
\hline \multirow{3}{*}{3} & 31 & 0.25 & 0 & 25 & 1 \\
\hline & 32 & 0.25 & 0 & 50 & 1 \\
\hline & 33 & 0.25 & 0 & 75 & 1 \\
\hline \multicolumn{6}{|c|}{ PARAMETRIZAR NÚMERO DE FILAS DEL RAJON } \\
\hline \multirow{3}{*}{1} & 34 & 0.25 & 0 & 50 & 1 \\
\hline & 35 & 0.25 & 0 & 50 & 2 \\
\hline & 36 & 0.25 & 0 & 50 & 3 \\
\hline \multirow{3}{*}{2} & 37 & 0.25 & 0 & 50 & 1 \\
\hline & 38 & 0.25 & 0 & 50 & 2 \\
\hline & 39 & 0.25 & 0 & 50 & 3 \\
\hline \multirow{3}{*}{3} & 40 & 0.25 & 0 & 50 & 1 \\
\hline & 41 & 0.25 & 0 & 50 & 2 \\
\hline & 42 & 0.25 & 0 & 50 & 3 \\
\hline
\end{tabular}




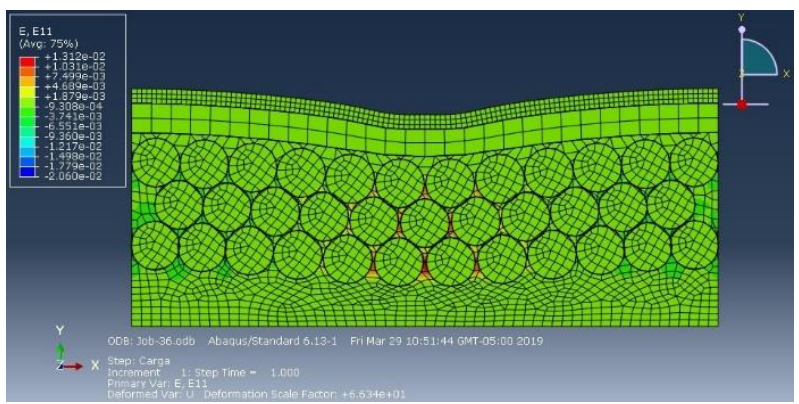

Figura 5. Diagrama de contornos de la deformación unitaria E11 (Ex) para modelación 36.

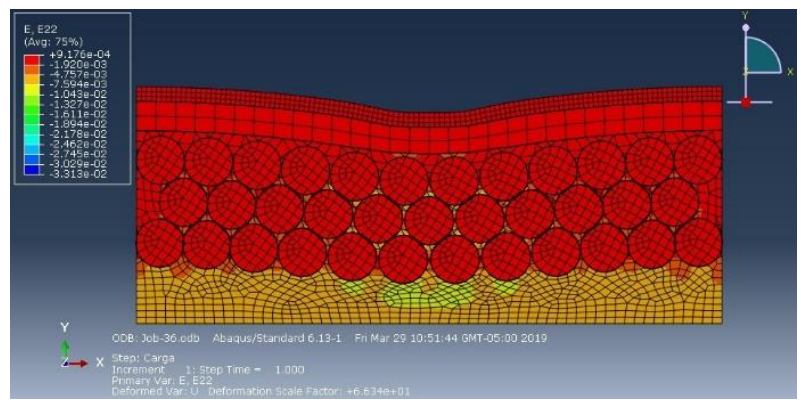

Figura 6. Diagrama de contornos de la deformación unitaria E22 (cy) para modelación 36.

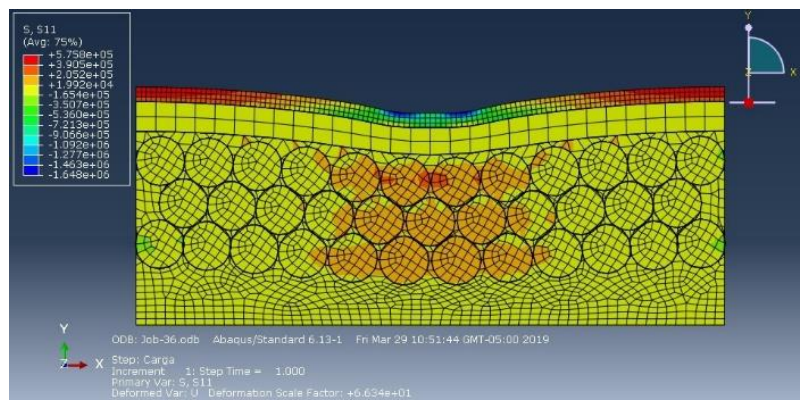

Figura 7. Diagrama de contornos del esfuerzo S11 ( $\sigma \mathrm{x})$ para modelación 36, valores de la escala en Pa.

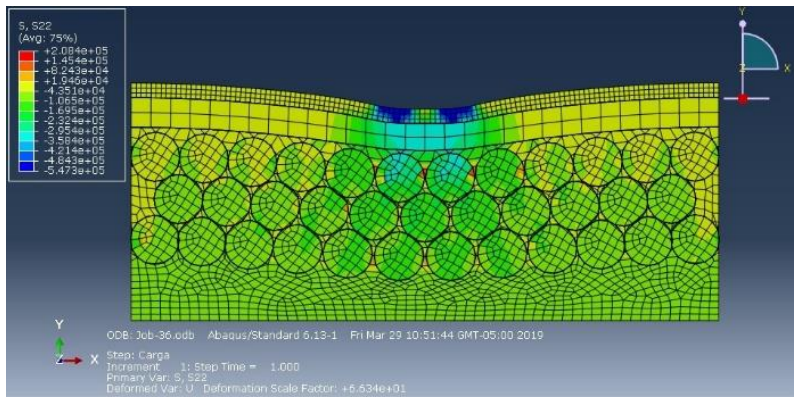

Figura 8. Diagrama de contornos del esfuerzo S22 ( $\sigma y)$ para modelación 36, valores de la escala en $\mathrm{Pa}$

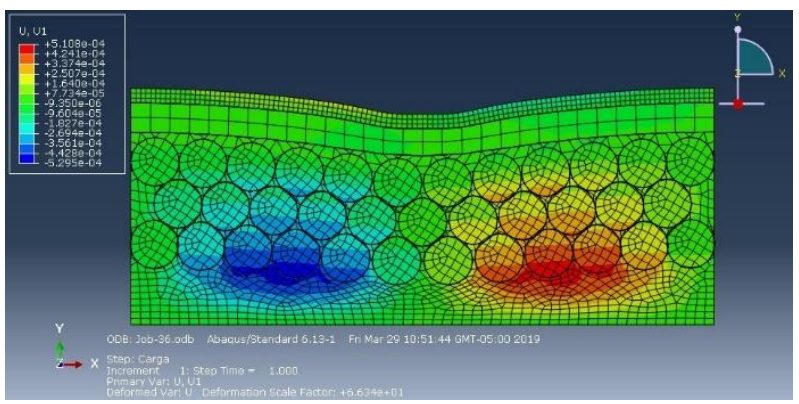

Figura 9. Diagrama de contornos del desplazamiento U11 ( $\delta \mathrm{x})$ para modelación 36 , valores de la escala en m.

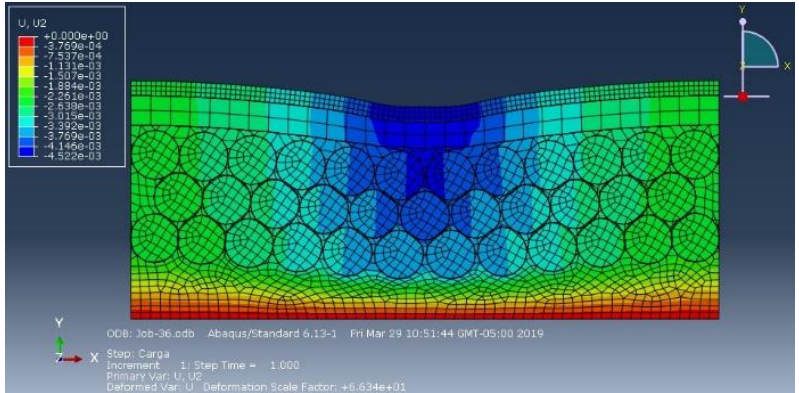

Figura 10. Diagrama de contornos del desplazamiento U22 ( $\delta$ y) para modelación 36, valores de la escala en m.

Las deformaciones unitarias, esfuerzos y desplazamientos resultados de la modelación se presentan en las siguientes graficas:

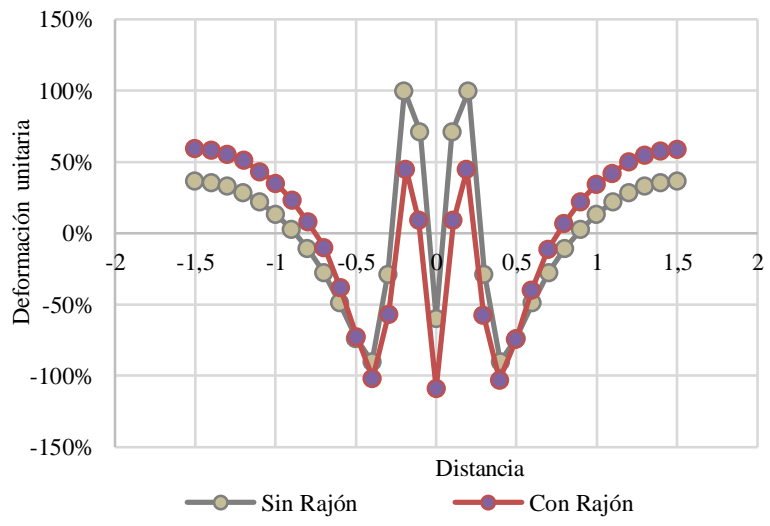

Figura 11. Deformación unitaria E11 en el eje x de la parte inferior de la carpeta $(\mathrm{Y}=1,3 \mathrm{~m}$, ver figura 4 - ejes de análisis) para modelación 36. 


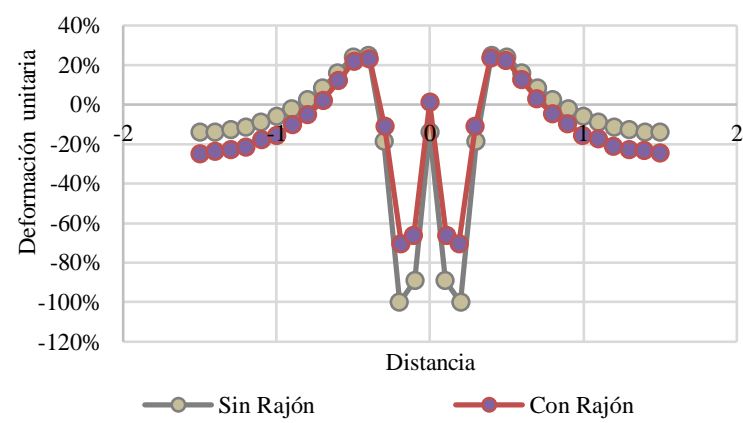

Figura 12. Deformación unitaria E22 en el eje x de la parte inferior de la carpeta $(\mathrm{Y}=1,3 \mathrm{~m})$ para modelación 36.

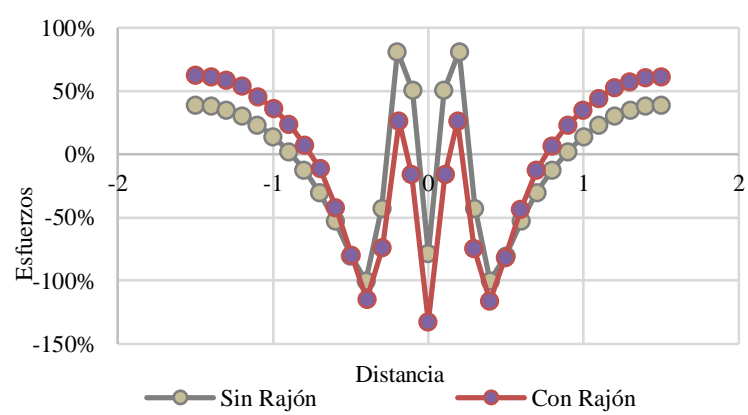

Figura 13. Esfuerzos $\mathrm{S} 11$ en el eje $\mathrm{x}$ de la parte inferior de la carpeta $(\mathrm{Y}=1,3 \mathrm{~m})$ para modelación 36 .

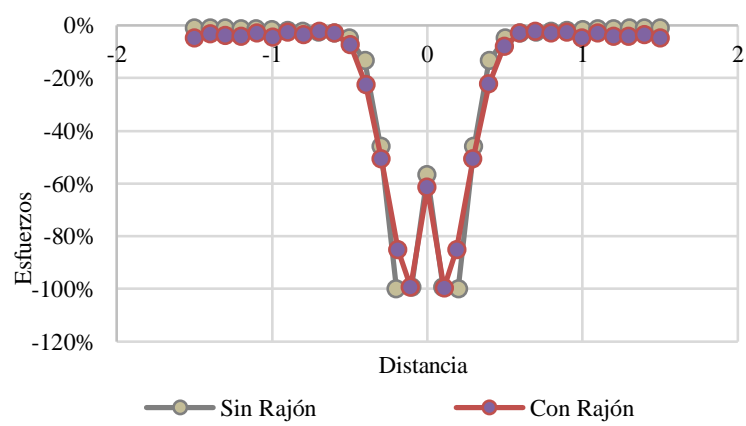

Figura 14. Esfuerzos $\mathrm{S} 22$ en el eje $\mathrm{x}$ de la parte inferior de la carpeta $(Y=1,3 \mathrm{~m})$ para modelación 36.

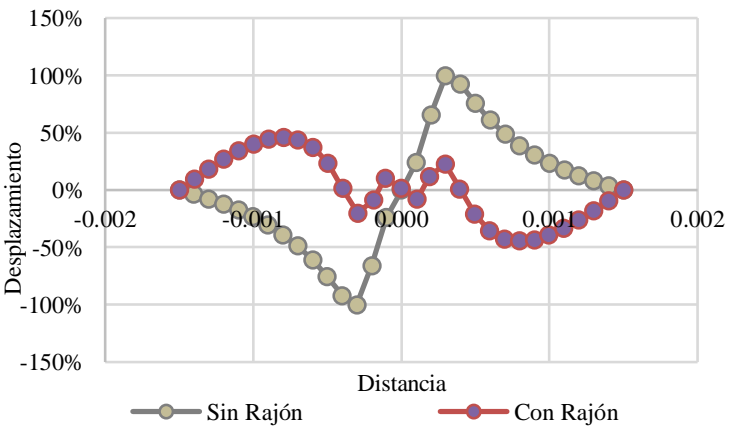

Figura 15. Desplazamientos U11 en el eje x de la parte inferior de la carpeta $(Y=1,3 \mathrm{~m})$ para modelación 36 .

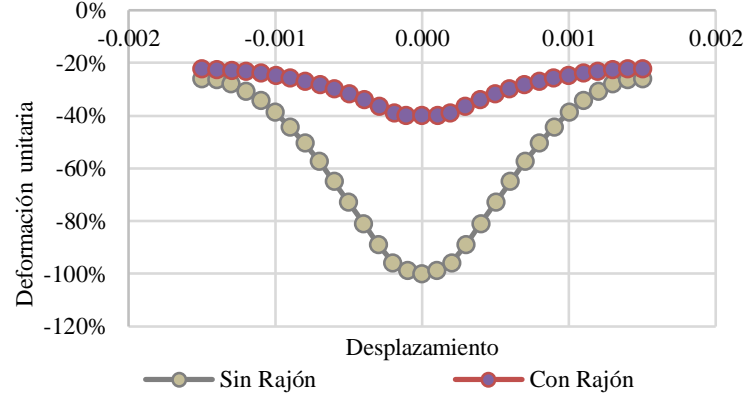

Figura 16. Desplazamientos U22 en el eje $\mathrm{x}$ de la parte inferior de la carpeta $(\mathrm{Y}=1,3 \mathrm{~m})$ para modelación 36.

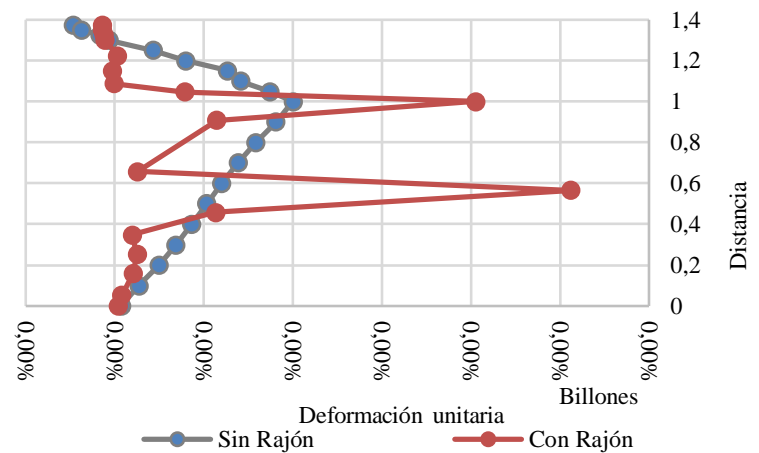

Figura 17. Deformación unitaria E11 en el eje Y que pasa por puntos de análisis para modelación 36 .

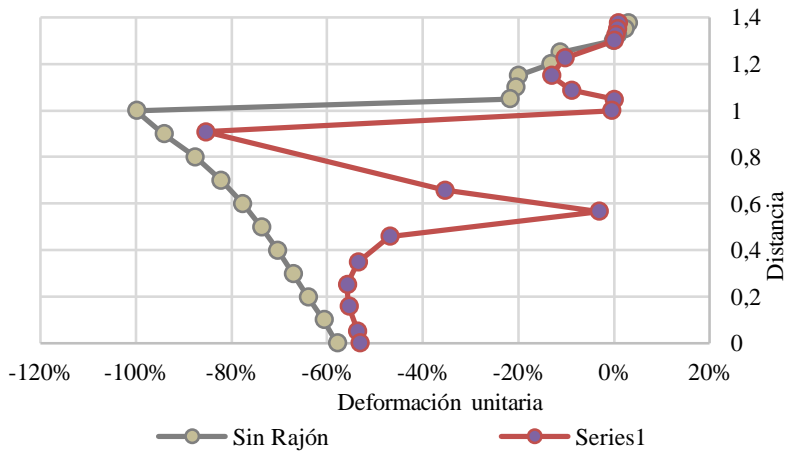

Figura 18. Deformación unitaria E22 en el eje Y que pasa por puntos de análisis para modelación 36.

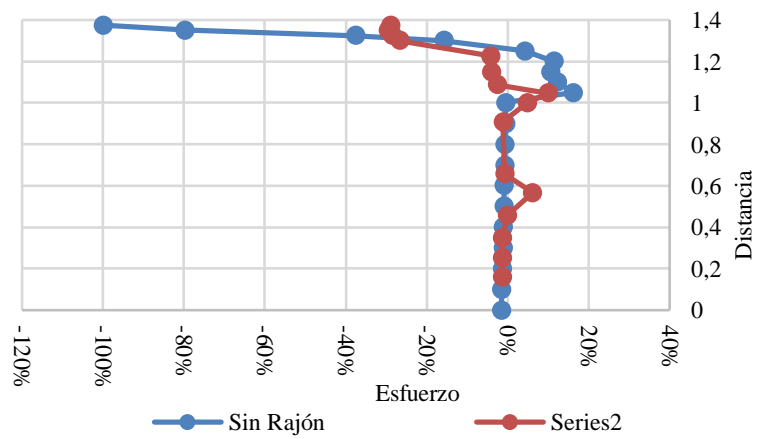

Figura 19. Esfuerzos $\mathrm{S} 11$ en el eje $\mathrm{Y}$ que pasa por puntos de análisis para modelación 36. 


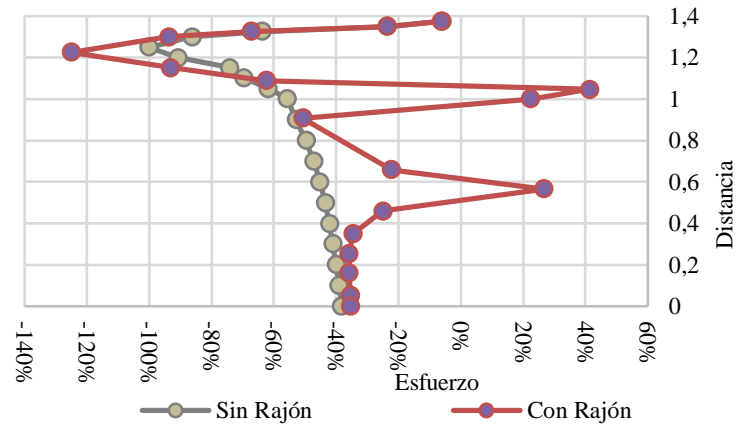

Figura 20. Esfuerzos S22 en el eje Y que pasa por puntos de análisis para modelación 36.

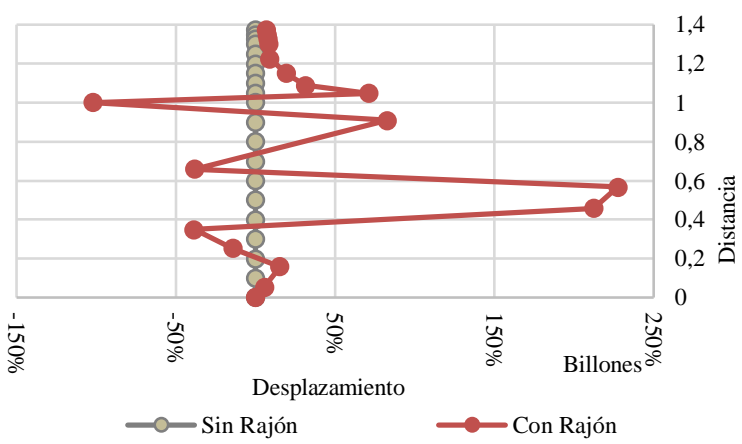

Figura 21. Desplazamientos U11 en el eje Y que pasa por puntos de análisis para modelación 36.

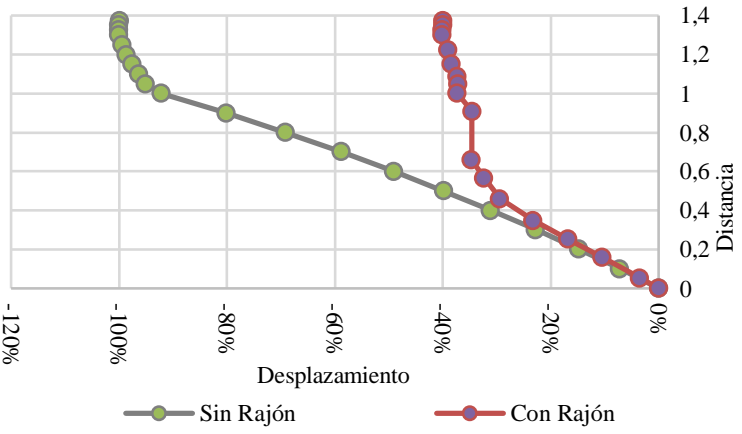

Figura 22. Desplazamientos U22 en el eje Y que pasa por puntos de análisis para modelación 36.

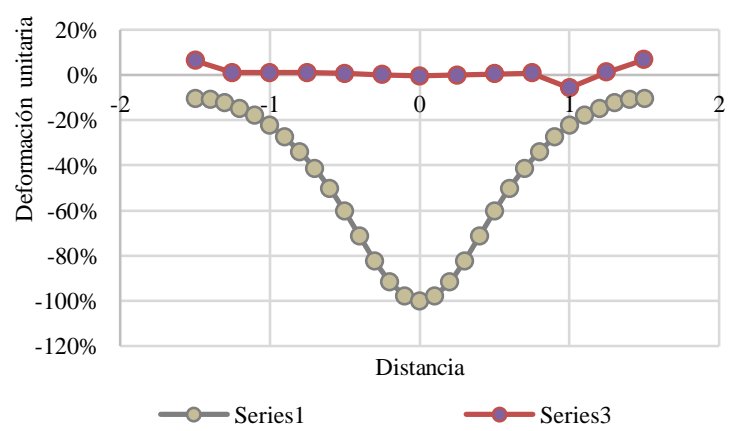

Figura 23. Deformaciones unitarias E22 en el eje X a nivel de subrasante N 1 m para modelación 36.

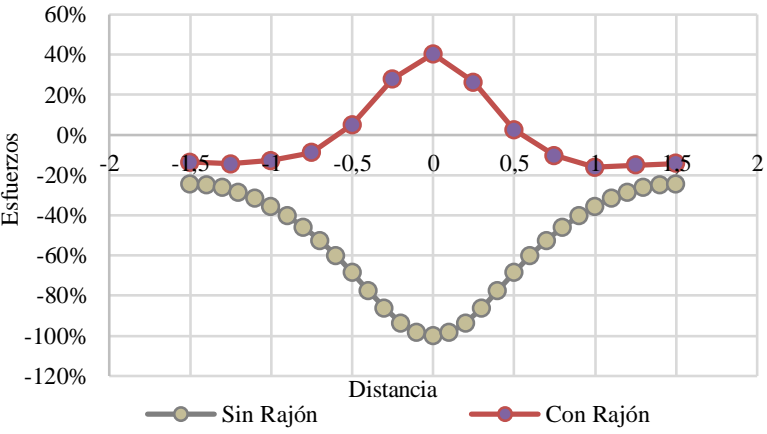

Figura 24. Esfuerzos $\mathrm{S} 22$ en el eje $\mathrm{X}$ a nivel de subrasante N 1 m para modelación 36.

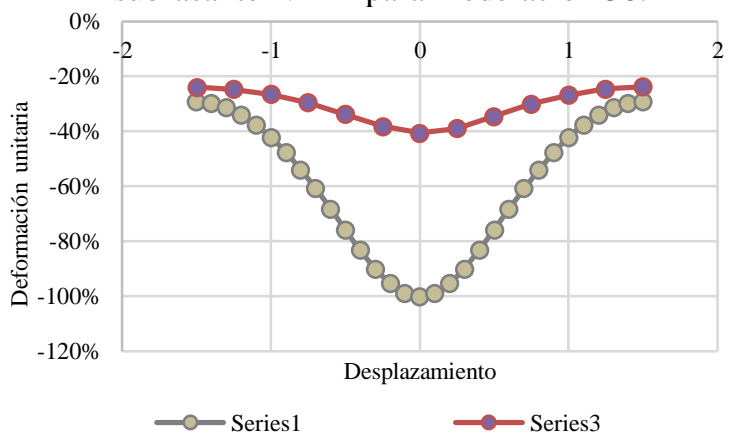

Figura 25. Desplazamiento U22 en el eje $\mathrm{X}$ a nivel de subrasante N 1 m para modelación 28.

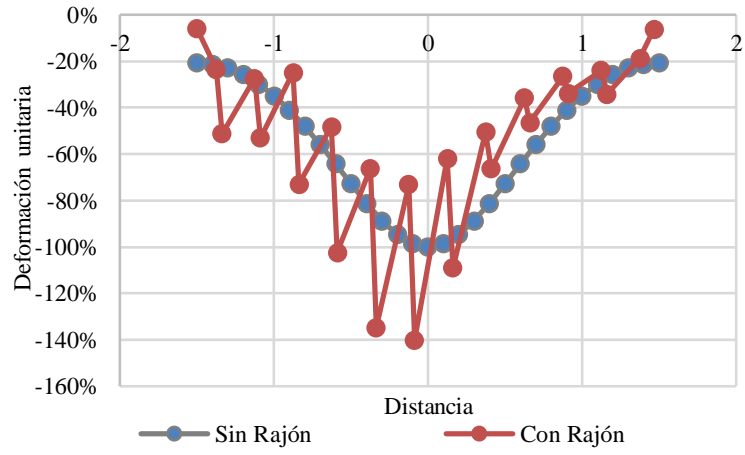

Figura 26. Deformaciones unitarias E22 en el eje X a nivel de subrasante $\mathrm{N} 0,7 \mathrm{~m}$ para modelación 36 .

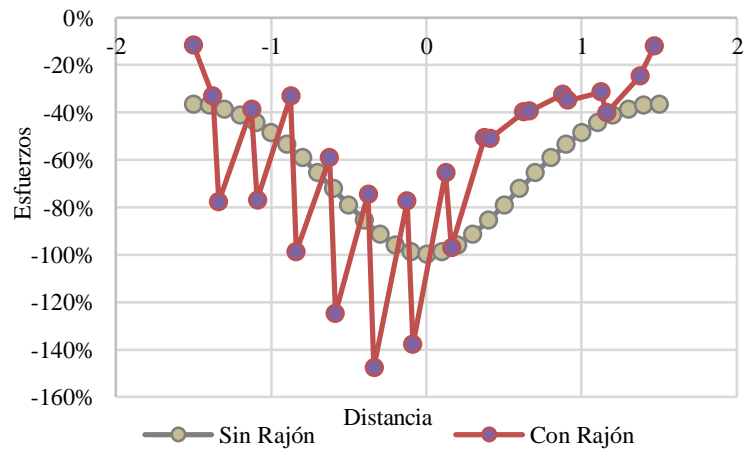

Figura 27. Esfuerzos $\mathrm{S} 22$ en el eje $\mathrm{X}$ a nivel de subrasante N 0,7 m para modelación 36 . 


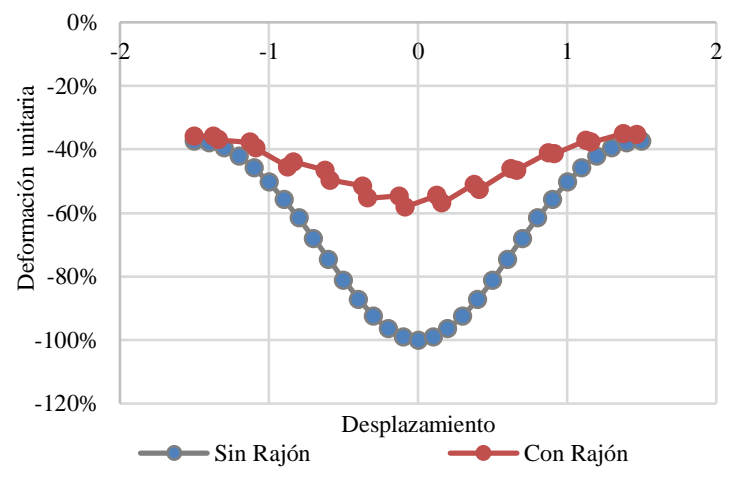

Figura 28. Desplazamiento U22 en el eje $\mathrm{X}$ a nivel de subrasante N 0,7 m para modelación 36.

\subsection{Comparaciones entre modelaciones mediante porcentajes, según los resultados obtenidos por el software de modelación}

En total, se realizó el análisis de 8 comparaciones, que al aplicarlas a las 42 modelaciones, arrojaron un total de 25 comparaciones, cada una con 18 gráficas comparativas del comportamiento de desplazamientos, deformaciones unitarias y esfuerzos; es decir, un total de 450 gráficas comparativas.

A continuación (véanse figura 29 a figura 46) se describen las comparaciones realizadas:

1. Variando CBR de subrasante $(1 \%, 2 \%, 3 \%)$.

2. Mismo CBR y variando diferentes diámetros.

3. Mismo diámetro, diferentes CBR (1\% $2 \% 3 \%)$.

4. Diferente espaciamiento, mismo CBR.

5. Mismo espaciamiento, diferente CBR.

6. Variando penetración de rajón, mismo CBR.

7. Misma penetración rajón, diferente CBR.

8. Variando número de filas de rajón, mismo CBR.

9. Igual número de filas de rajón, diferente CBR.

Se tienen las modelaciones comparativas y los resultados de análisis de las modelaciones de la 4 a la 42 con respecto a las modelaciones base. A continuación, se indica la comparación de las modelaciones de mismo CBR $3 \%$, diferente número de filas de rajón. Es importante notar que todas las diferencias porcentuales se amarran con respecto a la comparativa base de CBR $1 \%$ sin rajón, para tener punto de compración entre todos los resultados.

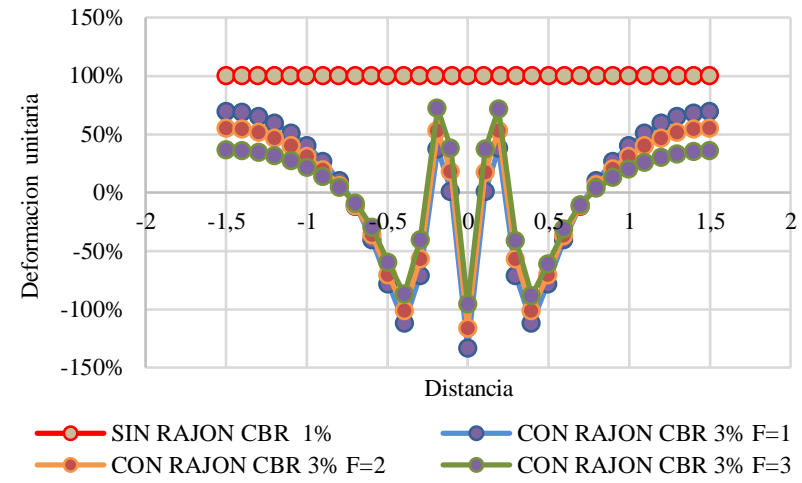

Figura 29. Comparación deformación unitaria E11 en el eje $\mathrm{X}$ de la parte inferior de la carpeta $(\mathrm{Y}=1,3 \mathrm{~m})$ para modelación 40, 41 y 42.

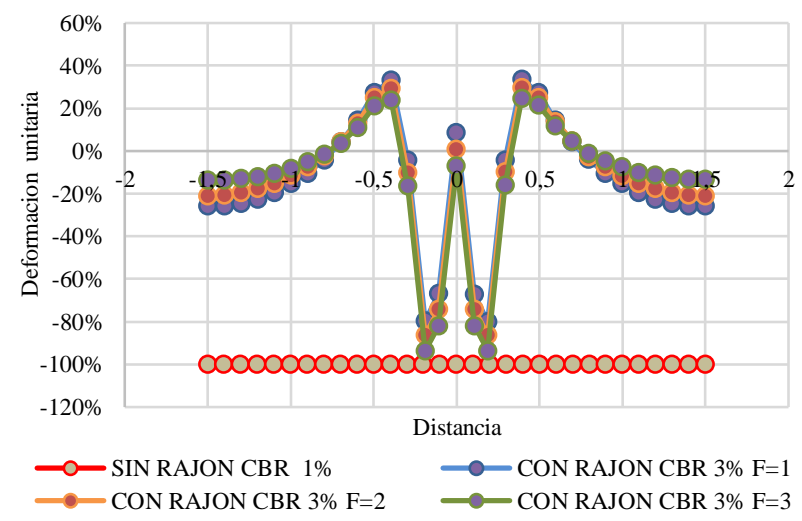

Figura 30. Comparación deformación unitaria E22 en el eje $\mathrm{X}$ de la parte inferior de la carpeta $(\mathrm{Y}=1,3 \mathrm{~m})$ para modelación 40, 41 y 42.

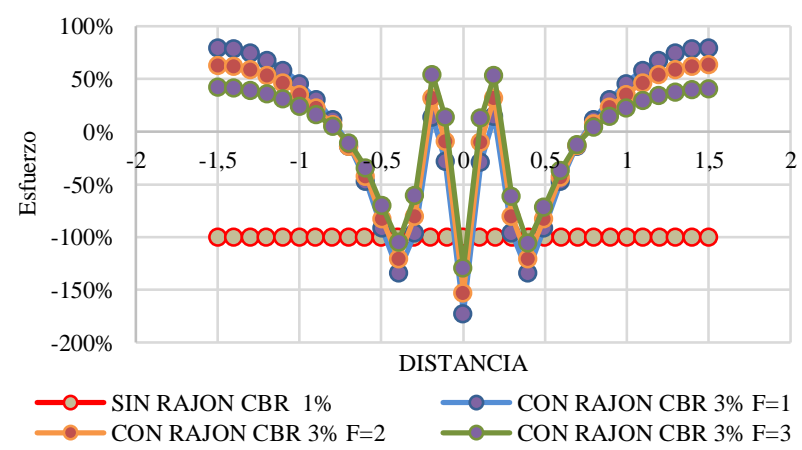

Figura 31. Comparación esfuerzos $\mathrm{S} 11$ en el eje $\mathrm{X}$ de la parte inferior de la carpeta $(\mathrm{Y}=1,3 \mathrm{~m})$ para modelación 40,41 y 42. 


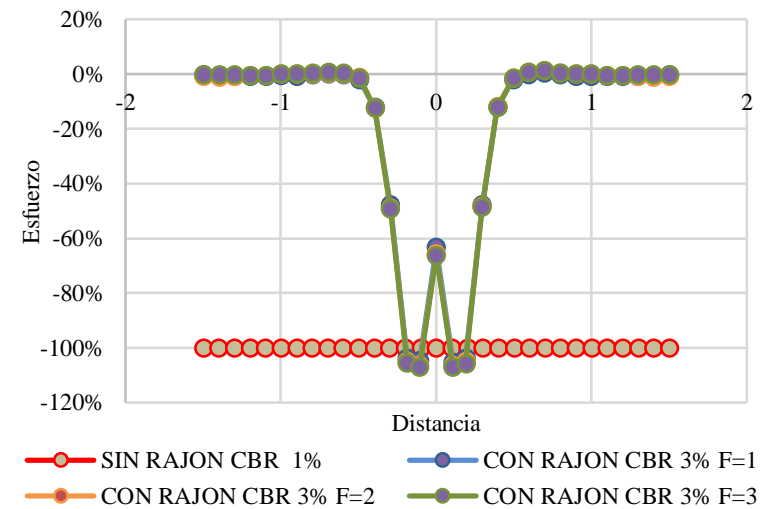

Figura 32. Comparación esfuerzos $\mathrm{S} 22$ en el eje $\mathrm{X}$ de la parte inferior de la carpeta $(\mathrm{Y}=1,3 \mathrm{~m})$ para modelación 40,41 y 42 .

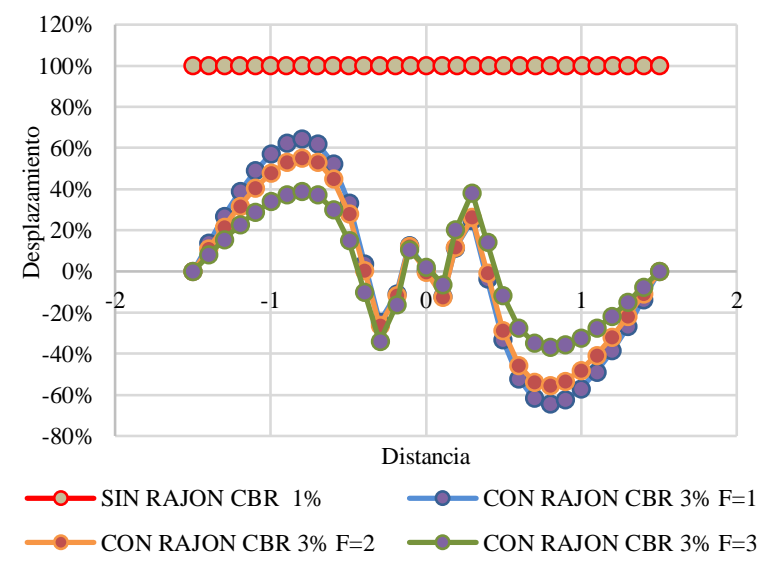

Figura 33. Comparación desplazamientos U11 en el eje $\mathrm{X}$ de la parte inferior de la carpeta $(\mathrm{Y}=1,3 \mathrm{~m})$ para modelación 40, 41 y 42.

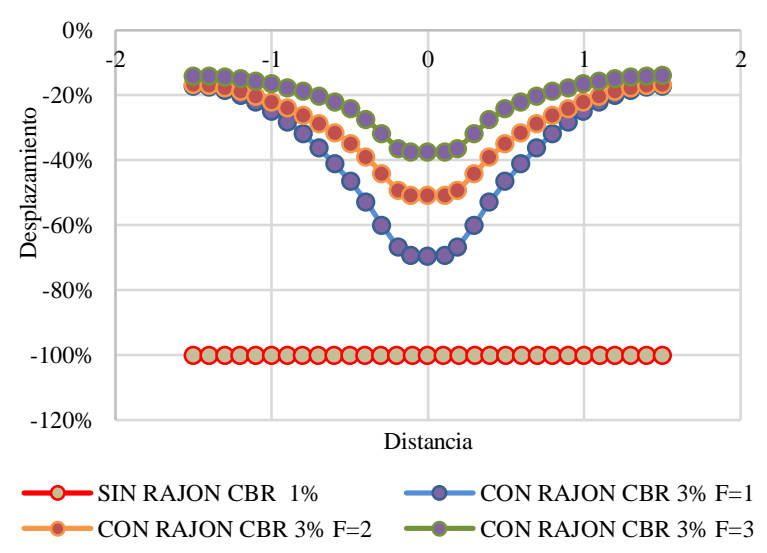

Figura 34. Comparación desplazamientos U22 en el eje $\mathrm{X}$ de la parte inferior de la carpeta $(\mathrm{Y}=1,3 \mathrm{~m})$ para modelación 40, 41 y 42.

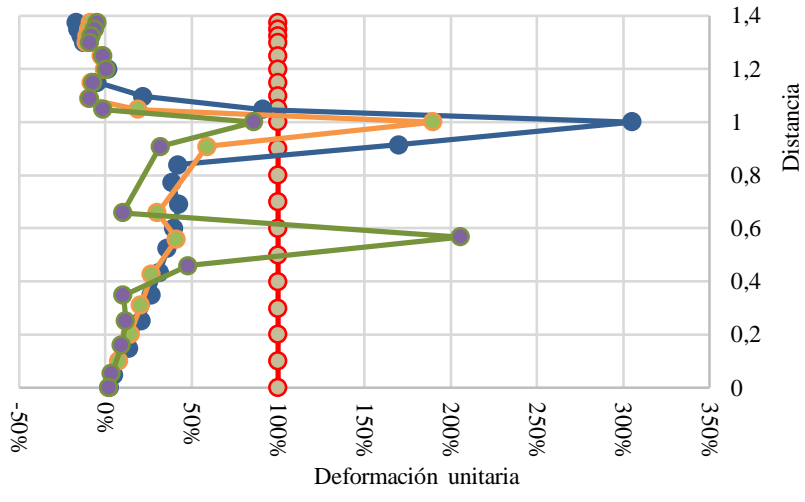

$\longrightarrow$ SIN RAJON CBR $1 \% \quad-$ CON RAJON CBR $3 \% \mathrm{~F}=1$ CON RAJON CBR $3 \% \mathrm{~F}=2 \longrightarrow$ CON RAJON CBR $3 \% \mathrm{~F}=3$

Figura 35. Comparación deformación unitaria E11 en el eje Y que pasa por puntos de análisis para modelación 40,41 y 42 .

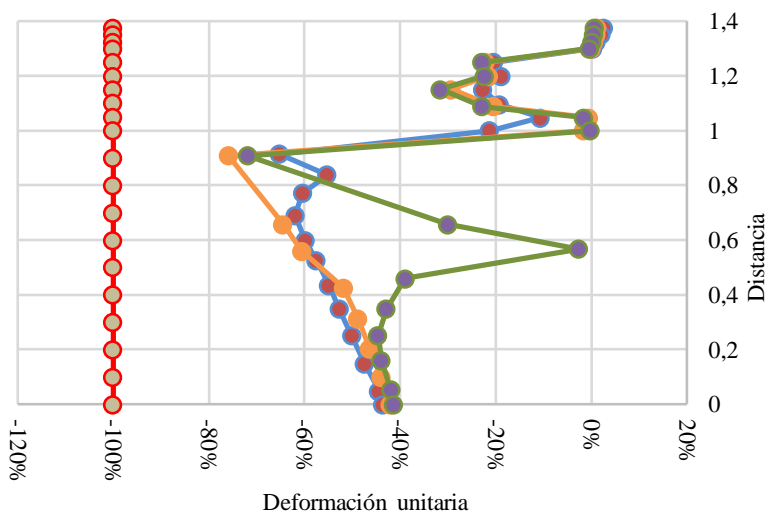

- SIN RAJON CBR $1 \%$
- CON RAJON CBR 3\% F=2 CON RAJON CBR 3\% F=1

Figura 36. Comparación deformación unitaria E22 en el eje Y que pasa por puntos de análisis para modelación 40,41 y 42 .

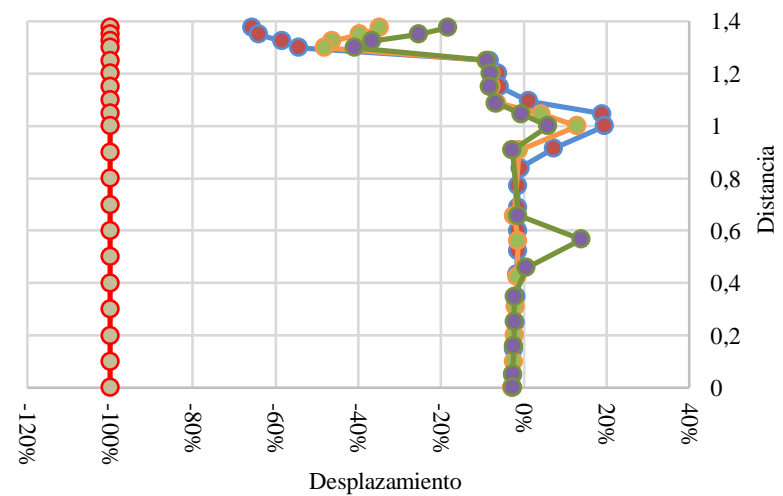

- SIN RAJON CBR $1 \%$
- CON RAJON CBR 3\% F=2 CON RAJON CBR 3\% F=1

Figura 37. Comparación esfuerzos S11 en el eje Y que pasa por puntos de análisis para modelación 40, 41 y 42. 


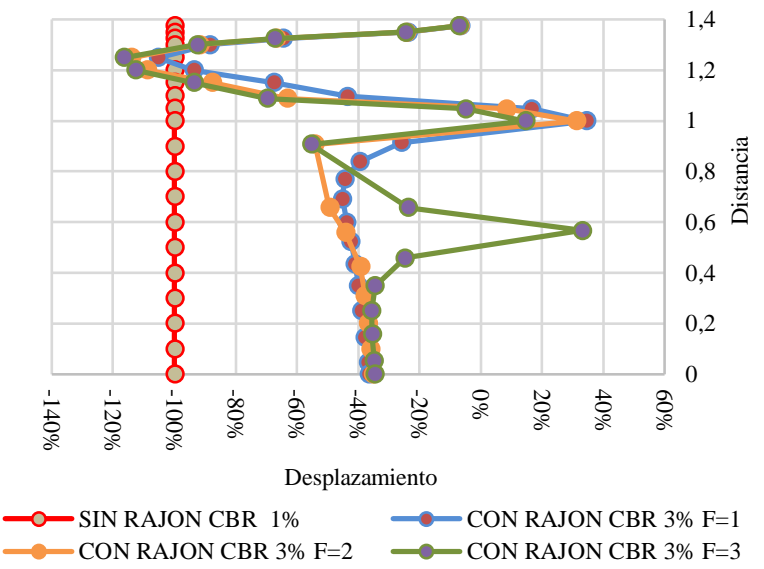

Figura 38. Comparación esfuerzos S22 en el eje Y que pasa por puntos de análisis para modelación 40, 41 y 42.

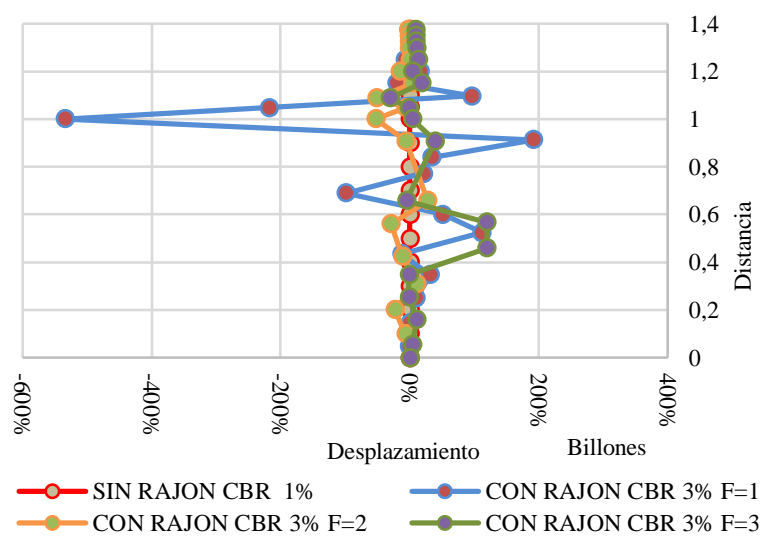

Figura 39. Comparación desplazamientos U11 en el eje Y que pasa por puntos de análisis para modelación 40, 41 y 42.

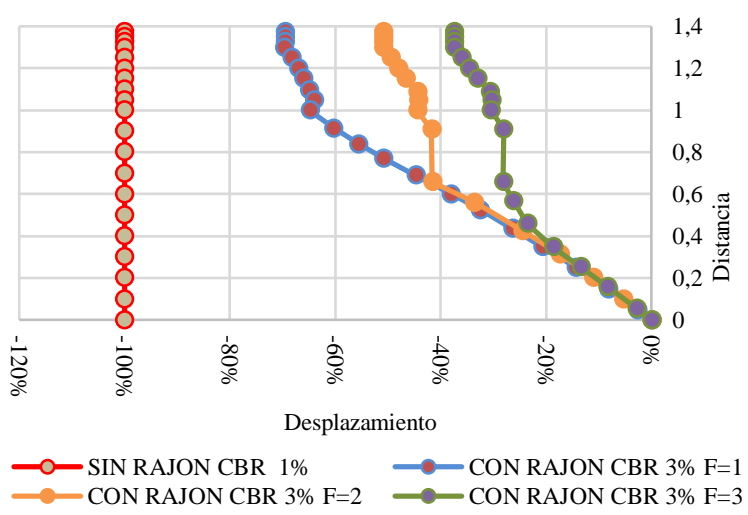

Figura 40. Comparación desplazamientos U22 en el eje Y que pasa por puntos de análisis para modelación 40 , 41 y 42.

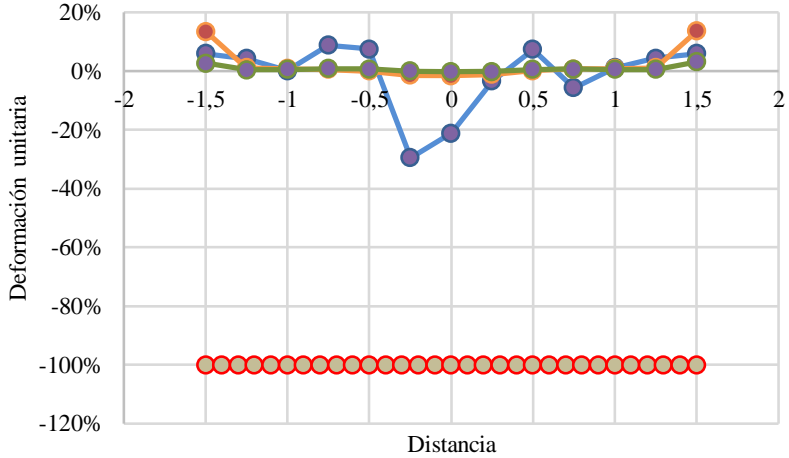

$\longrightarrow$ SIN RAJON CBR $1 \%$ CON RAJON CBR 3\% F=1
- CON RAJON CBR 3\% F=2 CON RAJON CBR 3\% F=3

Figura 41. Comparación deformación unitaria E22 en el eje X nivel de subrasante N 1 m, para modelación 40, 41 y 42.

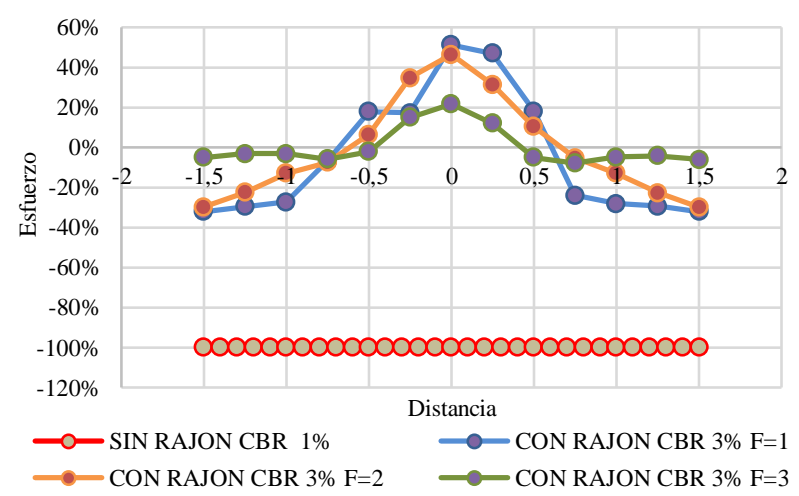

Figura 42. Comparación esfuerzos S22 en el eje X nivel de subrasante N 1 m, para modelación 40, 41 y 42.

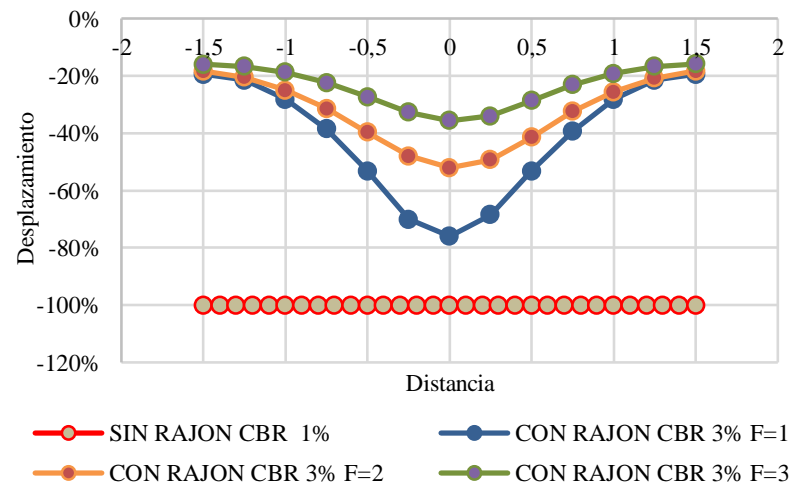

Figura 43. Comparación desplazamientos U22 en el eje X nivel de subrasante N 1 m, para modelación 40, 41 y 42. 


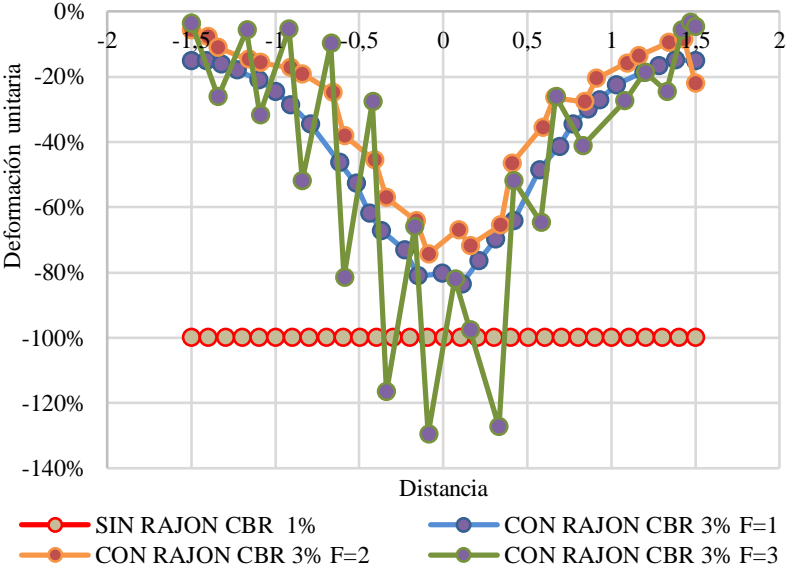

Figura 44. Comparación deformación unitaria E22 en el eje X nivel de subrasante N 0,7 m, para modelación 40, 41 y 42.

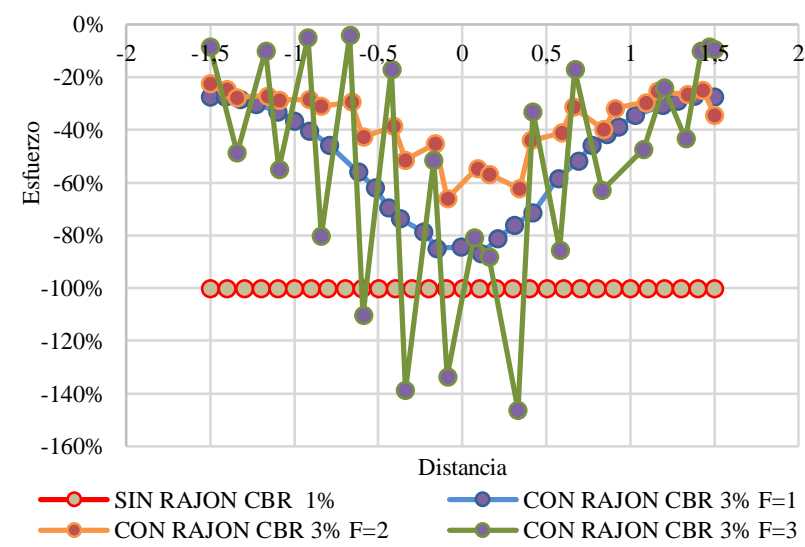

Figura 45. Comparación esfuerzos $\mathrm{S} 22$ en el eje $\mathrm{X}$ nivel de subrasante $\mathrm{N}$ 0,7 m, para modelación 40, 41 y 42 .

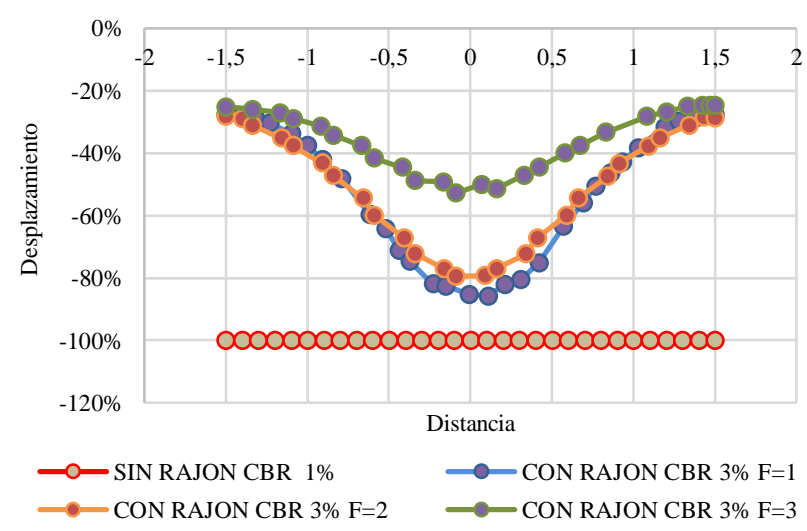

Figura 46. Comparación desplazamientos U22 en el eje $\mathrm{X}$ nivel de subrasante $\mathrm{N} 0,7 \mathrm{~m}$, para modelación 40, 41 y 42 .

\section{Conclusiones}

\subsection{Enfoque subrasante}

Considerando que para efectos de diseños de pavimentos flexibles se contempla la deflexión a nivel de subrasante [19], el cual en las modelaciones ejecutadas se trata del nivel $1,0 \mathrm{~m}$ de la subrasante (parte superior de la subrasante), y que el presente trabajo se enfoca principalmente en el comportamiento de la subrasante al adicionarle rajón, observando los resultados del análisis comparativo de la modelaciones, a continuación, se describen las conclusiones de las deformaciones en la parte superior de la subrasante:

- Al aumentar el diámetro del rajón, disminuyen los desplazamientos U22 y las deformaciones unitarias E22 en la subrasante, observándose que para la subrasante con CBR $1 \%$ disminuyen hasta un $31 \%$ y $91 \%$, respectivamente, cuando el diámetro de rajón es igual a $0,35 \mathrm{~m}$.

- Al disminuir el espaciamiento entre partículas de rajón, disminuyen los desplazamientos U22 y las deformaciones unitarias E22 en la subrasante, observándose que para la subrasante con CBR $1 \%$ disminuyen hasta un $22 \%$ y $81 \%$, respectivamente, cuando el rajón está configurado sin separación.

- Al aumentar la penetración del rajón en la capa de subrasante, disminuyen los desplazamientos U22 y las deformaciones unitarias E22 en la subrasante, observándose que para la subrasante con CBR $1 \%$ disminuyen hasta un $31 \%$ y $77 \%$, respectivamente, cuando el rajón se configura con penetración del $75 \%$.

- Al aumentar el número de filas de rajón en la capa de subrasante, disminuyen los desplazamientos U22 y las deformaciones unitarias E22 en la subrasante, observándose que para la subrasante con CBR $1 \%$ disminuyen hasta un $59 \%$ y $94 \%$, respectivamente, cuando se configuran 3 filas de rajón. Es de resaltar que para los casos de subrasantes con CBR $2 \%$ y CBR $3 \%$, las deformaciones unitarias E22 en la subrasante disminuyen hasta un $100 \%$ para la configuración correspondiente a tres filas de rajón.

- Es notorio que en el mejoramiento con rajón se presentan esfuerzos de tensión S22 de la parte superior de la subrasante en casi todos los casos. 


\subsection{Generalidades}

- La estructura de pavimento con tres filas de rajón presenta un mejor comportamiento en general respecto a desplazamientos, deformaciones y esfuerzos. En otras palabras, el módulo de elasticidad del material se incrementa, y, para la misma carga, se tienen menores deformaciones y solicitaciones a la estructura del pavimento.

- La estructura de pavimento con filas de rajón embebidas a $75 \%$ de su diámetro en la subrasante presentan mejor comportamiento en cuanto a desplazamientos, deformaciones y esfuerzos.

- En general, la estructura de pavimento con menor separación entre rajones presenta mejor comportamiento en cuanto a desplazamientos, deformaciones y esfuerzos.

- En general, la estructura de pavimento conformada por rajones de mayor diámetro presenta mejor comportamiento en cuanto a desplazamientos, deformaciones y esfuerzos.

- Se observó, de manera general, en el eje vertical (en el centro de la estructura), que los parámetros en estudio (deformación, esfuerzo y desplazamiento) fueron afectados sustancialmente en el área de influencia directa del rajón.

- Para lograr apreciar mejor las tendencias para futuros trabajos y/o investigaciones, se recomienda aumentar el número de modelaciones, aumentando el análisis de estructuras consideradas.

- Teniendo en cuenta resultados críticos (máximos), los esfuerzos S11 y S22 de la parte inferior de la carpeta asfáltica aumentan con la utilización de rajón.

- Teniendo en cuenta resultados críticos (máximos), los esfuerzos S22 de la parte superior de la subrasante (nivel $1 \mathrm{~m}$ ), en muchos casos, presentan comportamientos a tracción, situación que se presenta entre más aumente el diámetro de rajón, como también entre más disminuya la separación entre estos.
- Para cada CBR se presenta mejor comportamiento (disminución de deformaciones, esfuerzos y desplazamientos) a medida que se aumenta el

diámetro del rajón, a excepción de los parámetros S11 y S22 de la parte inferior de la carpeta asfáltica, donde aumentan los esfuerzos.

- Para cada CBR se presenta mejor comportamiento (disminución de deformaciones, esfuerzos y desplazamientos) a menor espaciamiento entre rajón, a excepción de los parámetros S11 y S22 de la parte inferior de la carpeta asfáltica, donde aumentan los esfuerzos a menor espaciamiento.

- Para cada CBR se presenta mejor comportamiento de desplazamientos, deformaciones, y esfuerzos en la parte inferior de la carpeta asfáltica a medida que aumenta la penetración del rajón.

- Para cada CBR se presenta mejor comportamiento de deformaciones, esfuerzos y desplazamientos en el nivel $0,7 \mathrm{~m}$ (véase figura 4 ) de subrasante a medida que disminuye la penetración del rajón.

- Para cada CBR, a mayor número de filas de rajón, el comportamiento de los distintos parámetros (deformación, tracción y desplazamiento) mejora (disminuyen).

- Los esfuerzos S22 de la parte superior de la subrasante (nivel $1 \mathrm{~m}$ ) para cada CBR, diámetro $0,25 \mathrm{~m}$ y penetración $50 \%$, tienen en general comportamientos a tensión.

- Rajón de 0,15 m, separación de 0 m y penetración del $50 \%$ con CBR $1 \%$ o CBR $2 \%$ o CBR $3 \%$ es la única estructura que presenta disminución de esfuerzos S22 en la parte inferior de la carpeta asfáltica.

- Rajón de 0,15 m, separación de 0 m y penetración del $50 \%$ con CBR $1 \%$ o CBR $2 \%$ o CBR $3 \%$ es la única estructura que presenta disminución de esfuerzos S22 en la parte superior de la subrasante. 


\subsection{Lineamientos de diseño}

- Se recomienda escoger los diámetros de material de sobretamaño (rajón) más grande posibles, estos demuestran el mejor impacto en el mejoramiento.

- Es posible colocar el mejoramiento con rajón existiendo separación entre partículas, para condiciones de diferente CBR; entre menor sea la separación del rajón, se obtiene mejor comportamiento de los parámetros en estudio (deformaciones, esfuerzos y desplazamientos).

- Se deberá colocar el rajón al mayor porcentaje de penetración posible, esto hace que se presente mejor comportamiento (disminución de deformaciones, esfuerzos y deformaciones).

- A mayor número de filas, se presenta mejor comportamiento (disminución de deformaciones, esfuerzos y deformaciones).

\subsection{Metodología de diseño básica con rajón para pavimentos}

- Determinar la granulometría del material disponible (rajón) — determinar $\mathrm{D}_{50}$ - a partir de un lote que se espera que traiga una o varias volquetas promedio en el proyecto. El material de rajón deberá cumplir las normas técnicas que apliquen al proyecto.

- Seguir los lineamientos de diseño establecido para seleccionar el material.

- Determinar empíricamente en campo qué penetración del rajón se espera $(25 \%, 50 \%, 75 \%, 100 \%)$.

- Determinar empíricamente el número de filas a colocar (mínimo una y se recomienda que máximo tres en la primera prueba), se podrá en el refinamiento posterior agregar o quitar filas.

- Hacer la modelación respectiva en elementos finitos, siguiendo los pasos mostrados en este artículo.

- Realizar el diseño de pavimento y determinar el mejoramiento y el impacto en el pavimento.

- Refinar el diseño del mejoramiento con rajón de acuerdo con los resultados obtenidos.

\section{Recomendaciones}

Se podrá continuar este trabajo, ampliando el número de modelaciones, $\mathrm{y}$, finalmente, con las parametrizaciones realizar ábacos de diseño, simplificando la metodología de diseño propuesta.

\section{Agradecimientos}

A la Universidad del Cauca por proporcionar el tiempo, el espacio y los equipos para realizar esta investigación.

\section{Referencias}

[1] Instituto Nacional de Vías, Manual de diseño de pavimentos asfálticos para vías con bajos volúmenes de tránsito. Bogotá D. C., Colombia: Gobierno de Colombia, 2007.

[2] Instituto Nacional de Vías, Normas de ensayo y especificaciones generales de construcción. Bogotá D. C., Colombia: INVÍAS, 2013.

[3] Instituto Desarrollo Urbano, Especificaciones técnicas de construcción. Bogota D.C., Colombia: Alcaldía Mayor de Bogotá D. C., 2005.

[4] Instituto Desarrollo Urbano, Manual de especificaciones técnicas de diseño y construcción de parques y escenarios públicos de Bogotá D.C. Bogota D.C, Colombia: Alcaldía Mayor de Bogotá D. C., 2005.

[5] J. P. Giroud, J. Han, "Desing method for geogridreinforced unpaved roads. i development of desing method", Journal of Geotechnical and Geoenvironmental Engineering, vol. 130, no. 8, pp. 775-786, 2004, doi: 10.1061/(ASCE)10900241(2004)130:8(775)

[6] J. Giroud, J. Han, "Desing metho for geogridreinforced unpaved roads. ii calibration and applications", Journal of Geotechnical and Geoenvironmental Engineering, vol. 130, no. 8, pp. 787-797, 2004, doi: 10.1061/(ASCE)10900241(2004)130:8(787)

[7] W. R. Calderón, M. R. P. Muñoz, "Desarrollo de un modelo de elementos finitos para el diseño racional de pavimentos", Tecnura, vol. 19, no. 7, pp. 25-37, 2005. 
[8] J. A. A. Pabón, Diapositivas sobre estabilización de subrasantes, Civilgeeks.com - Ingenieria y Construcción, [En línea]. Disponible en: http://civilgeeks.com/2013/11/01/diapositivas-sobreestabilizacion-de-subrasantes/

[9] D. A. C. Cruz, B. C. Hormaza, J. Tristancho, "Dynamic analysis of axes single, tandem, and tridem on viscoelastic pavements, with a model in the time domain", tesis de grado, Repositorio Universidad de los Andes, 2014

[10] W. R. Calderón, M. R. P Muñoz, "Desarrollo de un modelo de elementos finitos para el diseño racional de pavimentos", Tecnura, vol. 9, no. 17, pp. 25-37. 2005.

[11] E. O. Duarte, "Simulación de una estructura de pavimento apoyada en una subrasante arcillosa fracturada por desecación", tesis de grado, Universidad Santo Tomás, 2017.

[12] Abaqus/CAE, Abaqus analysis user's manual, Abaqus versión 6.1, [En línea]. Disponible en: http://130.149.89.49:2080/v6.7/books/usb/default.htm ?startat=pt08ch28s05aus112.html.

[13] M.C. Duarte, P. V. Sierra, "Estudio del comportamiento de un material de subrasante típico de Bogotá estabilizado con un sistema de geoceldas ante la aplicación de ciclos de carga y descarga mediante pruebas de laboratorio", tesis de grado, Pontificia Universidad Javeriana, 2011.

[14] American Association of State Highway and Transportation Officials, Guide for design of pavement structures. Washington, D.C, USA: AASHTO, 1993.

[15] C. L. Naranjo, J. A. A. Pabón, Manual de diseño de pavimentos de concreto: para vías con bajos, medios y altos volúmenes de tránsito. Medellín : ICPC; 2008

[16] Límites de pesos y dimensiones en los vehículos de transporte terrestre automotor de carga por carretera , para su operación normal en la red vial a nivel nacional, Ministerio de Transporte, Republica de Colombia. Resolución No 004100, 2004.
[17] C. A. B. Bastidas, E. C. Barreto, Caracterización dinamica de materiales viales y su aplicación al diseño racional de paviemntos fléxibles. Popayán, 1993.

[18] NCHRP, National Cooperative Highway Researh Program, MEPDG, Guide for mechanistic-empirical design of new and rehabilitated pavement structures. Illinois, 2004.

[19]. P. Leiva, L. Loria, S. A. Navas, “Comparación entre modelación de respuestas de pavimentos flexibles con analisis de multicapa elastica, y elemento finito", en XVI Congreso Ibero-Latinoamericano do Asfalto, Rio de Janeiro, 2011. 